\title{
POISSON CAPACITIES
}

\author{
E. B. Dynkin And S. E. Kuznetsov
}

\begin{abstract}
Poisson capacities appeared, first, in the theory of removable boundary singularities for solutions of semilinear elliptic differential equations. It seems that these capacities should play a key role for solving the problem: to describe all positive solutions of the equation $L u=u^{\alpha}$ with $\alpha>1$ in a smooth domain. We establish two inequalities for the Poisson capacities which might be useful for this task.
\end{abstract}

\section{Introduction}

1.1. Motivation. A Poisson capacity $\mathrm{CP}_{\alpha}(\Gamma)$ was introduced in [DK96] as a tool for a study of semilinear elliptic differential equations $L u=u^{\alpha}$. It was proved that, if $D$ is a bounded smooth domain, then a closed subset $\Gamma$ of $\partial D$ is a removable singularity for positive solutions of $L u=u^{\alpha}$ in $D$ if and only if $\mathrm{CP}_{\alpha}(\Gamma)=0$. First, this was conjectured in [Dyn94]. In the case $\alpha=2$, the conjecture was proved by Le Gall [Le95] who used a different definition of capacity (not applicable to $\alpha \neq 2$ ). Le Gall's capacity $\mathrm{Cap}^{\partial}$ has the same class of null sets as $\mathrm{CP}_{\alpha}$.

The present paper is motivated by a recent work by Mselati [Ms02] who proved that positive solutions of $\Delta u=u^{2}$ are characterized uniquely by their fine boundary traces introduced in [DK98]. ${ }^{1}$ A key part of Mselati's investigation is establishing bounds for certain solutions (associated with $\Gamma$ ) in terms of $\operatorname{Cap}^{\partial}(\Gamma)$. A challenging problem is to get a similar result in the case $\alpha \neq 2$ by using Poisson capacities. This note may be considered as a step in this direction.

1.2. Capacity Cap. Fix a constant $\alpha>1$, a bounded smooth domain $D$ of class $C^{2, \lambda}$ in $\mathbb{R}^{d}$ and a second order uniformly elliptic differential operator $L$ in $D$.

Denote by $\mathcal{M}(\Gamma)$ the set of all finite measures on $\Gamma$ and put $\mu \in \mathcal{P}(\Gamma)$ if, in addition, $\mu(\Gamma)=1$. There exists a positive function $k(x, y)$ (called the Poisson

Received August 25, 2002.

2000 Mathematics Subject Classification. Primary 31C15, Secondary 35J65, 60J60.

Key words and phrases. Poisson capacities, removable boundary singularities, semilinear elliptic PDEs.

Partially supported by National Science Foundation Grant DMS-0204237 and DMS9971009.

${ }^{1}$ This result was obtained in [DK98] for the so-called $\sigma$-moderate solutions. Mselati proved that all solutions are $\sigma$-moderate. 
kernel of $L$ in $D$ ) such that the formula

$$
h_{\mu}(x)=\int_{\partial D} k(x, y) \mu(d y)
$$

establishes a 1-1 correspondence between $\mathcal{M}(\partial D)$ and the set of all positive solutions $h$ of the equation $L h=0$ in $D$. We define a Poisson capacity of a Borel set $\Gamma \subset \partial D$ by the formula

$$
\operatorname{Cap}(\Gamma)=\sup _{\nu \in \mathcal{P}(\Gamma)} H(\nu)^{-1}
$$

where

$$
H(\mu)=\int_{D} \operatorname{dist}(y, \partial D) h_{\mu}(y)^{\alpha} d y
$$

$\left[\operatorname{Cap}(\Gamma)=\mathrm{CP}_{\alpha}(\Gamma)^{\alpha}\right.$ where $\mathrm{CP}_{\alpha}$ is the capacity used in [Dy02]. A subscript $\alpha$ can be dropped since $\alpha$ is fixed.]

We denote by $C$ constants depending only on $D, L$ and $\alpha$ (their values can vary even within one line). We indicate explicitely the dependence on any additional parameter. For instance, we write $C_{\kappa}$ for a constants depending on a parameter $\kappa$ (besides a possible dependence on $D, L, \alpha)$. An upper bound of $\operatorname{Cap}(\Gamma)$ is given by:

Theorem 1.1. For all $\Gamma$,

$$
\operatorname{Cap}(\Gamma) \leq C \operatorname{diam}(\Gamma)^{\gamma_{+}}
$$

where

$$
\begin{gathered}
\operatorname{diam}(\Gamma)=\sup _{x, y \in \Gamma}|x-y| \\
\gamma=d \alpha-d-\alpha-1 \quad \text { and } \gamma_{+}=\gamma \vee 0 .
\end{gathered}
$$

1.3. Capacities $\operatorname{Cap}_{x}$. Put

$$
H_{x}(\mu)=\int_{D} g(x, y) h_{\mu}(y)^{\alpha} d y
$$

where $g(x, y)$ is the Green function of $L$ in $D$. To every $x \in D$ there corresponds a capacity on $\partial D$ defined by the formula

$$
\operatorname{Cap}_{x}(\Gamma)=\sup _{\nu \in \mathcal{P}(\Gamma)} H_{x}(\nu)^{-1}
$$

The second theorem establishes a lower bound for $\mathrm{Cap}_{x}$ in terms of Cap.

The values $\alpha<(d+1) /(d-1)$ are called subcritical and the values $\alpha \geq$ $(d+1) /(d-1)$ are called supercrtical.

Theorem 1.2. Suppose that $L$ is an operator of divergence form (1.13) and $d \geq 3$. Put

$$
\varphi(x)=\operatorname{dist}(x, \partial D) \operatorname{dist}(x, \Gamma)^{-d} .
$$


If $\alpha$ is subcritical, then

$$
\operatorname{Cap}_{x}(\Gamma) \geq C \varphi(x)^{-1} \operatorname{Cap}(\Gamma) .
$$

for all $\Gamma$ and $x$.

If $\alpha$ is supercritical, then, for every $\kappa>0$ there exists a constant $C_{\kappa}$ such that

$$
\operatorname{Cap}_{x}(\Gamma) \geq C_{\kappa} \varphi(x)^{-1} \operatorname{Cap}(\Gamma)
$$

for all $\Gamma$ and $x$ subject to the condition

$$
\operatorname{dist}(x, \Gamma) \geq \kappa \operatorname{diam}(\Gamma) .
$$

[An analog of formula (1.11) with Cap replaced by $\mathrm{Cap}^{\partial}$ follows from formula (3.34) in [Ms02] in the case $L=\Delta, \alpha=2, d \geq 4$ and $\kappa=4$.]

1.4. On operator $L$ and domain $D$. We consider an operator

$$
L u(x)=\sum_{i, j=1}^{d} a_{i j}(x) \frac{\partial^{2}}{\partial x_{i} \partial x_{j}} u(x)+\sum_{i=1}^{d} b_{i}(x) \frac{\partial}{\partial x_{i}} u(x)
$$

in a domain $D$ in $\mathbb{R}^{d}$. Without loss of generality we can put $a_{i j}=a_{j i}$. We assume that

\subsection{A. ${ }^{2}$ There exists a constant $\beta>0$ such that}

$$
\sum a_{i j}(x) t_{i} t_{j} \geq \beta \sum t_{i}^{2} \quad \text { for all } x \in D, t_{1}, \ldots, t_{d} \in \mathbb{R} .
$$

1.4.B. All coefficients $a_{i j}(x)$ and $b_{i}(x)$ are Hölder continuous in $\bar{D}$ with exponent $\lambda$ and Hölder's coefficient $\Lambda$.

We assume that the domain $D$ is smooth (more precicely, $D$ is a domain of class $C^{2, \lambda}$ ) which means that $\partial D$ can be straightened near every point $x \in \partial D$. To define straightening, we consider a half-space $E_{+}=\left\{x=\left(x_{1}, \ldots, x_{d}\right): x_{d}>\right.$ $0\}=\mathbb{R}^{d-1} \times \mathbb{R}_{+}$and its boundary $E_{0}=\left\{x=\left(x_{1}, \ldots, x_{d}\right): x_{d}=0\right\}$. We assume that, for every $x \in \partial D$, there exists a ball $B(x, \varepsilon)=\{y:|x-y|<\varepsilon\}$ and a diffeomorphism $\psi_{x}$ of class $C^{2, \lambda}$ from $B(x, \varepsilon)$ onto a domain $\tilde{D} \subset \mathbb{R}^{d}$ such that $\psi(B(x, \varepsilon) \cap D) \subset E_{+}$and $\psi(B(x, \varepsilon) \cap \partial D) \subset E_{0}$. We say that $\psi_{x}$ straightens the boundary in $B(x, \varepsilon)$.

In Theorem 1.2 we restrict ourself to operators of divergence form

$$
L u(x)=\sum_{i, j=1}^{d} \frac{\partial}{\partial x_{i}} a_{i j}(x) \frac{\partial}{\partial x_{j}} u(x) .
$$

\footnotetext{
${ }^{2}$ The property 1.4.A is called uniform ellipticity and $\beta$ is called the ellipticity coefficient of $L$.
} 
1.5. Bounds for $g(x, y)$ and $k(x, y)$. In the proofs of Theorems 1.1 and 1.2 we use the following bounds for the Poisson kernel (see, e.g. [Maz75], Lemma 6 and the Appendix B in [Dy02]):

$$
C^{-1} \rho(x)|x-y|^{-d} \leq k(x, y) \leq C \rho(x)|x-y|^{-d}
$$

where

$$
\rho(x)=\operatorname{dist}(x, \partial D) .
$$

[It follows from (1.14) that, if $\mathrm{Cap}^{\prime}$ and $\mathrm{Cap}^{\prime \prime}$ are the Poisson capacities associated with operators $L^{\prime}$ and $L^{\prime \prime}$, then $\operatorname{Cap}^{\prime}(\Gamma) / \operatorname{Cap}^{\prime \prime}(\gamma) \leq C$ where $C$ is a constant independent of $\Gamma$.]

In the proof of Theorem 1.2 we also use bounds for the Green function:

$$
\begin{gathered}
g(x, y) \leq C|x-y|^{2-d}, \\
g(x, y) \leq C \rho(x)|x-y|^{1-d}, \\
g(x, y) \leq C \rho(x) \rho(y)|x-y|^{-d} .
\end{gathered}
$$

established in [GrW] for operators $L$ of divergence form and $d \geq 3$.

\section{Upper bound for $\operatorname{Cap}(\Gamma)$}

2.1. Capacity $\widehat{\mathbf{C a p}}$. To prove Theorem 1.1 we introduce a capacity on the set $E_{0}$ associated with the kernel

$$
\hat{k}(x, y)=r(x)|x-y|^{-d}, x \in E_{+}, y \in E_{0}
$$

where

$$
r(x)=\operatorname{dist}\left(x, E_{0}\right)=x_{d}
$$

Put

$$
\mathbb{E}=\left\{x=\left(x_{1}, \ldots, x_{d}\right): 0<x_{d}<1\right\},
$$

To every measure $\nu \in \mathcal{P}\left(E_{0}\right)$ there corresponds a function

$$
\hat{h}_{\nu}(x)=\int_{E_{0}} \hat{k}(x, y) \nu(d y)
$$

on $E_{+}$. Put

$$
\hat{H}(\nu)=\int_{\mathbb{E}} r(x) \hat{h}_{\nu}(x)^{\alpha} d x
$$

and

$$
\hat{H}(\nu, B)=\int_{B} r(x) \hat{h}(x)^{\alpha} d x
$$

for $B \subset E_{+}$. Note that

$$
\hat{k}(x / t, y / t)=t^{d-1} \hat{k}(x, y) \text { for all } t>0 .
$$


To every $\nu \in \mathcal{P}\left(E_{0}\right)$ there corresponds a measure $\nu_{t}$ defined by the formula $\nu_{t}(B)=\nu(t B)$. We have

$$
\int_{E_{0}} f(y) \nu_{t}(d y)=\int_{E_{0}} f(y / t) \nu(d y)
$$

for every function $f$ and therefore

$$
\hat{h}_{\nu_{t}}(x / t)=\int_{E_{0}} \hat{k}(x / t, y) \nu_{t}(d y)=\int_{E_{0}} \hat{k}(x / t, y / t) \nu(d y)=t^{d-1} \hat{h}_{\nu}(x) .
$$

Change of variables $x=t \tilde{x}$ and (2.4) yield

$$
\hat{H}\left(\nu_{t}\right)=t^{\gamma} \hat{H}(\nu, t \mathbb{E}) .
$$

If $t \geq 1$, then $t \mathbb{E} \supset \mathbb{E}$ and we have

$$
\hat{H}\left(\nu_{t}\right) \geq t^{\gamma} \hat{H}(\nu) .
$$

We introduce a capacity on $E_{0}$ by the formula

$$
\widehat{\operatorname{Cap}}(\Gamma)=\sup _{\nu \in \mathcal{P}(\Gamma)} \hat{H}(\nu)^{-1}
$$

Lemma 2.1. If $\operatorname{diam}(\Gamma) \leq 1$, then

$$
\widehat{\operatorname{Cap}}(\Gamma) \leq C_{d}(\operatorname{diam}(\Gamma))^{\gamma}
$$

The constant $C_{d}$ depends only on the dimension d. (It is equal to $\widehat{\operatorname{Cap}}(U)$ where $U=\left\{x \in E_{0}:|x|<1\right\}$.

Proof. Since $\widehat{\mathrm{Cap}}$ is translation invariant, we can assume that $0 \in \Gamma$. Let $t=$ $\operatorname{diam}(\Gamma)^{-1}$. Since $t \Gamma \subset U$, we have

$$
\widehat{\operatorname{Cap}}(t \Gamma) \leq \widehat{\operatorname{Cap}}(U) .
$$

Since $\nu \rightarrow \nu_{t}$ is a 1-1 mapping from $\mathcal{P}(t \Gamma)$ onto $\mathcal{P}(\Gamma)$, we get

$$
\widehat{\operatorname{Cap}}(\Gamma)=\sup _{\nu_{t} \in \mathcal{P}(\Gamma)} \hat{H}\left(\nu_{t}\right)^{-1}=\sup _{\nu \in \mathcal{P}(t \Gamma)} \hat{H}\left(\nu_{t}\right)^{-1} .
$$

Therefore, by (2.6) and (2.5),

$$
\widehat{\operatorname{Cap}}(\Gamma) \leq t^{-\gamma} \widehat{\operatorname{Cap}}(t \Gamma)
$$

and (2.8) implies (2.7).

\subsection{Two lemmas.}

Lemma 2.2. Suppose $D \subset \mathbb{E}, 0 \in \Gamma \subset \partial D \cap E_{0}$ and put $A=\partial D \cap E_{+}$, $B_{\lambda}=\{x \in \mathbb{E}:|x|<\lambda\}$. If $\operatorname{dist}(\Gamma, A)>2 \lambda$, then $B_{\lambda} \subset D$ and $r(x)=\rho(x)$ for $x \in B_{\lambda}$. 
Proof. If $x \in B_{\lambda}$, then $r(x) \leq|x|<\lambda$. If $x \in B_{\lambda}$ and $y \in A$, then $|x-y| \geq$ $|y|-|x|>\lambda$ because $|y|>\operatorname{dist}(y, \Gamma)>\operatorname{dist}(A, \Gamma)>2 \lambda$. Hence $\operatorname{dist}(x, A) \geq \lambda$ which implies that $B_{\lambda} \subset D$.

For $x \in D \cup \partial D, \rho(x)=\operatorname{dist}\left(x, D^{c}\right), r(x)=\operatorname{dist}\left(x, E_{+}^{c}\right)$ and therefore $\rho(x) \leq$ $r(x)$. Put $\tilde{A}=\partial D \backslash A$. Let $x \in B_{\lambda}$. Since $\rho(x)=\operatorname{dist}(x, \tilde{A}) \wedge \operatorname{dist}(x, A)$, the equality $r(x)=\rho(x)$ holds because $\operatorname{dist}(x, \tilde{A}) \geq r(x)$ (since $\tilde{A} \subset E_{0}$ ) and $\operatorname{dist}(x, A) \geq \lambda>r(x)$.

Lemma 2.3. There exists a constant $C$ (depending only on $\lambda$ ) such that

$$
\hat{H}\left(\nu, B_{\lambda}\right) \geq C \hat{H}(\nu)
$$

for all $\Gamma \ni 0$ such that $\operatorname{diam}(\Gamma)<\lambda / 2$ and for all $\nu \in \mathcal{P}(\Gamma)$.

Proof. If $x \in F_{\lambda}=E_{+} \backslash B_{\lambda}$ and $y \in \Gamma$, then $|y| \leq \operatorname{diam}(\Gamma)<\lambda / 2 \leq|x| / 2$ and therefore $|x-y|>|x|-|y| \geq|x| / 2$. This implies

$$
\hat{h}_{\nu}(x) \leq r(x) 2^{d}|x|^{-d}
$$

and

$$
\hat{H}\left(\nu, F_{\lambda}\right) \leq 2^{d \alpha} \int_{F_{\lambda}} r(x)^{\alpha+1}|x|^{-d \alpha} d x=C_{1}<\infty .
$$

On the other hand, if $x \in B_{\lambda}, y \in \Gamma$, then $|x-y| \leq|x|+|y| \leq 3 \lambda / 2$. Therefore $\hat{h}_{\nu}(x) \geq(3 \lambda / 2)^{-d} r(x)$ and

$$
\hat{H}\left(\nu, B_{\lambda}\right) \geq(3 \lambda / 2)^{-d \alpha} \int_{B_{\lambda}} r(x)^{\alpha+1} d x=C_{2}>0 .
$$

It follows from (2.10) and (2.11) that

$$
C_{1} \hat{H}\left(\nu, B_{\lambda}\right) \geq C_{1} C_{2} \geq C_{2} \hat{H}\left(\nu, F_{\lambda}\right)=C_{2}\left[\hat{H}(\nu)-\hat{H}\left(\nu, B_{\lambda}\right)\right]
$$

and (2.9) holds with $C=C_{2} /\left(C_{1}+C_{2}\right)$.

\subsection{Straightening of the boundary.}

Proposition 2.1. Suppose that $D$ is a bounded smooth domain. Then there exist strictly positive constants $\varepsilon, a, b$ such that, for every $x \in \partial D$ :

(a) The boundary can be straightened in $B(x, \varepsilon)$.

(b) The corresponding diffeomorphism $\psi_{x}$ satisfies the conditions

$$
\begin{gathered}
a^{-1}\left|y_{1}-y_{2}\right| \leq\left|\psi_{x}\left(y_{1}\right)-\psi_{x}\left(y_{2}\right)\right| \leq a\left|y_{1}-y_{2}\right| \quad \text { for all } y_{1}, y_{2} \in B(x, \varepsilon) \\
a^{-1} \operatorname{diam}(A) \leq \operatorname{diam}\left(\psi_{x}(A)\right) \leq a \operatorname{diam}(A) \quad \text { for all } A \subset B(x, \varepsilon) \\
a^{-1} \operatorname{dist}\left(A_{1}, A_{2}\right) \leq \operatorname{dist}\left(\psi_{x}\left(A_{1}\right), \psi_{x}\left(A_{2}\right)\right) \leq a \operatorname{dist}\left(A_{1}, A_{2}\right) \\
\quad \text { for all } A_{1}, A_{2} \subset B(x, \varepsilon) \\
b^{-1} \leq J_{x}(y) \leq b \quad \text { for all } y \in B(x, \varepsilon)
\end{gathered}
$$

where $J_{x}(y)$ is the Jacobian of $\psi_{x}$ at $y$. 
Diffeomorphisms $\psi_{x}$ can be chosen to satisfy additional conditions

$$
\psi_{x}(x)=0 \quad \text { and } \psi_{x}(B(x, \varepsilon)) \subset \mathbb{E} .
$$

Proof. The boundary $\partial D$ can be covered by a finite number of balls $B_{i}=$ $B\left(x_{i}, \varepsilon_{i}\right)$. The function $q(x)=\max _{i} \operatorname{dist}\left(x, B_{i}\right)$ is continuous and strictly positive on $\partial D$. Therefore $\varepsilon=\frac{1}{2} \min _{x} q(x)>0$. For every $x \in D$ we choose a ball $B_{i}$ which contains $x$ and we put

$$
\psi_{x}(y)=\psi_{x_{i}}(y) \quad \text { for } y \in B(x, \varepsilon) .
$$

This is a diffeomorphism straightening $\partial D$ in $B(x, \varepsilon)$.

Since the closure of $B(x, \varepsilon)$ is contained in $B_{i}, \psi_{x_{i}}$ is uniformly continuous on $B(x, \varepsilon)$. The inverse mapping is uniformly continuous on the image of $B(x, \varepsilon)$. Hence there exist constants $a_{i}>0$ such that

$$
a_{i}^{-1}\left|y_{1}-y_{2}\right| \leq\left|\psi_{x_{i}}\left(y_{1}\right)-\psi_{x_{i}}\left(y_{2}\right)\right| \leq a_{i}\left|y_{1}-y_{2}\right| \quad \text { for all } y_{1}, y_{2} \in B\left(x_{i}, \varepsilon_{i} / 2\right) \text {. }
$$

The condition (2.12) holds for $a=\max a_{i}$. The conditions (2.13) and (2.14) follow from (2.12). The condition (2.15) holds because $J_{x_{i}}$ is continuous and strictly positive on the closure of $B_{i}$.

By replacing $\psi_{x}(y)$ with $c\left[\psi_{x}(y)-\psi_{x}(x)\right]$ with is a suitable constant $c$, we get diffeomorphisms subject to (2.16) in addition to (2.12)-(2.15).

\subsection{Proof of Theorem 1.1.}

$1^{\circ}$. If $\gamma<0$, then (1.4) holds because $\operatorname{Cap}(\Gamma) \leq \operatorname{Cap}(\partial D)=C$. To prove (1.4) for $\gamma \geq 0$, it is sufficient to prove that, for some $\beta>0$, there is a constant $C_{1}$ such that

$$
\operatorname{Cap}(\Gamma) \leq C_{1} \operatorname{diam}(\Gamma)^{\gamma} \quad \text { if } \operatorname{diam}(\Gamma) \leq \beta .
$$

Indeed,

$$
\operatorname{Cap}(\Gamma) \leq C_{2} \operatorname{diam}(\Gamma)^{\gamma} \quad \text { if } \operatorname{diam}(\Gamma) \geq \beta
$$

with $C_{2}=\operatorname{Cap}(\partial D) \beta^{-\gamma}$.

$2^{\circ}$. Let $\varepsilon, a$ be the constants defined in Proposition 2.1 and let $\beta=\varepsilon / 2 \wedge 1$. Suppose that $\operatorname{diam}(\Gamma) \leq \beta$ and let $x \in \Gamma$. Consider a straightening $\psi_{x}$ of $\partial D$ in $B(x, \varepsilon)$ which satisfies conditions (2.16). Put $B=B(x, \varepsilon), \tilde{B}=$ $B(x, \varepsilon / 2)$. There exists a smooth domain $U$ such that $\tilde{B} \cap D \subset U \subset B \cap D$. Note that $\tilde{B} \cap \partial D \subset \partial U \cap \partial D \subset B \cap \partial D$. If $A=\partial U \cap B \cap D$, then $\operatorname{dist}(x, A) \geq \varepsilon / 2$ and $\operatorname{dist}(\Gamma, A) \geq \varepsilon / 2-\operatorname{diam}(\Gamma) \geq \varepsilon / 2-\beta$. Denote by $U^{\prime}, \Gamma^{\prime}, A^{\prime}$ the images of $U, \Gamma, A$ under $\psi_{x}$. By $(2.13), \operatorname{diam}\left(\Gamma^{\prime}\right) \leq \lambda_{1}=a \beta$ and $\operatorname{dist}\left(\Gamma^{\prime}, A\right) \geq \lambda_{2}=(\varepsilon / 2-\beta) / a$. If $\beta<\varepsilon\left(8 a^{2}+2\right)^{-1}$, then $\lambda_{1}<\lambda_{2} / 4$ and Lemmas 2.2 and 2.3 are applicable to $U^{\prime}, \Gamma^{\prime}$ and $\lambda=\frac{1}{2}\left(\lambda_{1}+\lambda_{2} / 4\right)$.

$3^{\circ}$. By (1.14) and (2.14), for every $y \in U, z \in \Gamma$,

$$
\begin{aligned}
& k(y, z) \geq C \operatorname{dist}(y, \partial D)|y-z|^{-d} \\
& \quad \geq C \operatorname{dist}(y, \partial U)|y-z|^{-d} \geq \frac{C}{a} \operatorname{dist}\left(y^{\prime}, \partial U\right)\left|y^{\prime}-z^{\prime}\right|^{-d}
\end{aligned}
$$


where $y^{\prime}=\psi_{x}(y), z^{\prime}=\psi_{x}(z)$. If $\nu^{\prime}$ is the image of $\nu \in \mathcal{P}(\Gamma)$ under $\psi_{x}$, then

$$
\int_{\Gamma} f\left[\psi_{x}(z)\right] \nu(d z)=\int_{\Gamma^{\prime}} f\left(z^{\prime}\right) \nu^{\prime}\left(d z^{\prime}\right)
$$

for every positive measurable function $f$. In particular,

$$
\int_{\Gamma}\left|y^{\prime}-\psi_{x}(z)\right|^{-d} \nu(d z)=\int_{\Gamma^{\prime}}\left|y^{\prime}-z^{\prime}\right|^{-d} \nu^{\prime}\left(d z^{\prime}\right) .
$$

By (2.17) and (2.18),

$$
\int_{\Gamma} k(y, z) \nu(d z) \geq C \operatorname{dist}\left(y^{\prime}, \partial U^{\prime}\right) \int_{\Gamma^{\prime}}\left|y^{\prime}-z^{\prime}\right|^{-d} \nu^{\prime}\left(d z^{\prime}\right)
$$

If $y^{\prime} \in B_{\lambda}$, then, by Lemma 2.2 , $\operatorname{dist}\left(y^{\prime}, \partial U^{\prime}\right)=r\left(y^{\prime}\right)$ and we have

$$
h_{\nu}(y)=\int_{\Gamma} k(y, z) \nu(d z) \geq C \int_{\Gamma^{\prime}} r\left(y^{\prime}\right)\left|y^{\prime}-z^{\prime}\right|^{-d} \nu^{\prime}\left(d z^{\prime}\right)=C \hat{h}_{\nu^{\prime}}\left[\psi_{x}(y)\right]
$$

By $(2.14), \operatorname{dist}(y, \partial D) \geq \operatorname{dist}(y, \partial U) \geq C \operatorname{dist}\left(y^{\prime}, \partial U^{\prime}\right)$ and therefore (1.3) and (2.19) imply

$$
H(\nu)=\int_{D} \operatorname{dist}(y, \partial D) h_{\nu}(y)^{\alpha} d y \geq C \int_{U} \operatorname{dist}\left(\psi_{x}(y), \partial U^{\prime}\right) \hat{h}_{\nu^{\prime}}\left[\psi_{x}(y)\right]^{\alpha} d y
$$

Note that

$$
\int_{U^{\prime}} f\left(y^{\prime}\right) d y^{\prime}=\int_{U} f\left[\psi_{x}(y)\right] J_{x}(y) d y
$$

and, if $f \geq 0$, then, by $(2.15)$,

$$
\int_{U^{\prime}} f\left(y^{\prime}\right) d y^{\prime} \leq b \int_{U} f\left[\psi_{x}(y)\right] d y .
$$

By taking $f\left(y^{\prime}\right)=\operatorname{dist}\left(y^{\prime}, \partial U^{\prime}\right) \hat{h}_{\nu^{\prime}}\left(y^{\prime}\right)^{\alpha}$, we get from $(2.20)$

$$
H(\nu) \geq C \int_{U^{\prime}} \operatorname{dist}\left(y^{\prime}, \partial U^{\prime}\right) \hat{h}_{\nu^{\prime}}\left(y^{\prime}\right)^{\alpha} d y^{\prime}
$$

By Lemma 2.2, $U^{\prime} \supset B_{\lambda}$ and $\operatorname{dist}\left(y^{\prime}, \partial U^{\prime}\right)=r\left(y^{\prime}\right)$ on $B_{\lambda}$. Hence

$$
H(\nu) \geq C \int_{B_{\lambda}} r\left(y^{\prime}\right) \hat{h}_{\nu^{\prime}}\left(y^{\prime}\right)^{\alpha} d y^{\prime}=C \hat{H}\left(\nu^{\prime}, B_{\lambda}\right)
$$

By Lemma 2.3, this implies $H(\nu) \geq C \hat{H}\left(\nu^{\prime}\right)$ and $\operatorname{Cap}(\Gamma) \leq C \widehat{\operatorname{Cap}}\left(\Gamma^{\prime}\right)$. The bound $\operatorname{Cap}(\Gamma) \leq C \operatorname{diam}(\Gamma)^{\gamma}$ follows from Lemma 2.1 and $(2.14)$. 


\section{Lower bound for $\operatorname{Cap}_{x}$}

3.1. Put

$$
\begin{aligned}
& \delta(x)=\operatorname{dist}(x, \Gamma), \quad D_{1}=\{x \in D: \delta(x)<\rho(x) / 2\}, \quad D_{2}=D \backslash D_{1} ; \\
& H_{x}(\nu, B)=\int_{B} g(x, y) h_{\nu}(y)^{\alpha} d y \quad \text { for } B \subset D
\end{aligned}
$$

and let

$$
U_{x}=\{y \in D:|x-y|<\delta(x) / 2\}, \quad V_{x}=\{y \in D:|x-y| \geq \delta(x) / 2\} .
$$

Theorem 1.2 follows from the following three lemmas.

Lemma 3.1. For all $\Gamma$, all $\nu \in \mathcal{P}(\Gamma)$ and all $x \in D$,

$$
H_{x}\left(\nu, V_{x}\right) \leq C \varphi(x) H(\nu) \text {. }
$$

Proof. By (1.14), for all $\nu \in \mathcal{P}(\Gamma)$ and all $y \in V_{x}$,

$$
h_{\nu}(y)=\int_{\Gamma} k(y, z) \nu(d z) \leq C \rho(y) \int_{\Gamma}|y-z|^{-d} \nu(d z) \leq C \varphi(x)
$$

and, by (1.18),

$$
H_{x}\left(\nu, V_{x}\right) \leq C \rho(x) \int_{V_{x}} \rho(y)|x-y|^{-d} h_{\nu}(y)^{\alpha} d y \leq C \varphi(x) H(\nu) .
$$

Lemma 3.2. For all $\Gamma$, all $\nu \in \mathcal{P}(\Gamma)$ and all $x \in D_{1}$,

$$
H_{x}\left(\nu, U_{x}\right) \leq C \varphi(x) H(\nu) \text {. }
$$

Proof. By the Harnack's inequality, if $h$ is harmonic in $D$ and if $|x-y| / \rho(x) \leq$ $r<1$, then

$$
\frac{1-r}{(1+r)^{d-1}} h_{\nu}(x) \leq h_{\nu}(y) \leq \frac{1+r}{(1-r)^{d-1}} h_{\nu}(x)
$$

(see, e.g. [GT98], Problem 2.61). If $x \in D_{1}$, then this inequality holds with $r=1 / 4$ for all $y \in U_{x}$. Therefore

$$
H_{x}\left(\nu, U_{x}\right) \leq C_{d} h_{\nu}(x)^{\alpha} \int_{U_{x}} g(x, y) d y
$$

and

$$
H(\nu) \geq C_{d} h_{\nu}(x)^{\alpha} \int_{U_{x}} \rho(y) d y .
$$

where $C_{d}$ depends only on $d$. By (1.17),

$$
\int_{U_{x}} g(x, y) d y \leq C \rho(x) \int_{U_{x}}|x-y|^{1-d} d y \leq C \delta(x) \rho(x) .
$$

Since $\rho(y) \geq \rho(x) / 2$ for $y \in U_{x}$, we have

$$
\int_{U_{x}} \rho(y) d y \geq \frac{1}{2} \rho(x) \int_{U_{x}} d y=C_{d} \rho(x) \delta(x)^{d} .
$$


Since $\delta \rho \leq \varphi \rho \delta^{d} / 2$, bound (3.4) follows from (3.5)-(3.8).

Lemma 3.3. For all $\Gamma$, all $\nu \in \mathcal{P}(\Gamma)$ and all $x \in D_{2}$,

$$
H_{x}\left(\nu, U_{x}\right) \leq C \varphi(x) \theta(x)^{-\gamma_{+}} H(\nu)
$$

where

$$
\theta(x)=\operatorname{dist}(x, \Gamma) / \operatorname{diam}(\Gamma) .
$$

Proof. If $\operatorname{diam}(\Gamma)=\lambda$, then, by (1.2), and Theorem 1.1,

$$
H(\nu)^{-1 / \alpha} \leq \operatorname{Cap}(\Gamma) \leq C \lambda^{\gamma+/ \alpha} .
$$

Hence,

$$
H(\nu) \geq C \lambda^{-\gamma+} .
$$

If $x \in D_{2}$ and $y \in U_{x}$, then $\delta(y) \geq \delta(x)-|x-y|>\delta(x) / 2$ and $\rho(y) \leq$ $\rho(x)+|x-y| \leq 2 \delta(x)+\delta(x) / 2=5 \delta(x) / 2$. For all $z \in \Gamma, y \in U_{x},|y-z| \geq$ $|z-x|-|y-x| \geq \delta(x) / 2$ and, by (1.14),

$$
k(y, z) \leq C \rho(y)|y-z|^{-d} \leq C \delta(x)^{1-d} .
$$

Therefore $h_{\nu}(y) \leq C \delta(x)^{1-d}$ and, by (1.17),

$$
H_{x}\left(\nu, U_{x}\right) \leq C \rho(x) \delta(x)^{(1-d) \alpha} \int_{U_{x}}|x-y|^{1-d} d y \leq C \varphi(x) \delta(x)^{-\gamma} .
$$

If $\gamma<0$, then $\delta(x)^{-\gamma} \leq \operatorname{diam}(D)^{-\gamma}=C$. If $\gamma \geq 0$, then $\gamma=\gamma_{+}$. Hence, the bound (3.9) follows from (3.10) and (3.11).

3.2. Proof of Theorem 1.2. Since $H_{x}(\nu)=H_{x}\left(\nu, V_{x}\right)+H_{x}\left(\nu, U_{x}\right)$, we get from Lemmas 3.1-3.3 that

$$
H_{x}(\nu) \leq C\left(1 \vee \theta(x)^{-\gamma_{+}}\right) \varphi(x) H(\nu)
$$

for all $\Gamma$, all $\nu \in \mathcal{P}(\Gamma)$ and all $x \in D$. By (1.2) and (1.8), this implies

$$
\operatorname{Cap}_{x}(\Gamma) \geq C\left(1 \vee \theta(x)^{\gamma_{+}}\right) \varphi(x)^{-1} \operatorname{Cap}(\Gamma)
$$

for all $\Gamma$ and all $x \in D$. If $\alpha$ is subcritical, then $\gamma<0$ and (3.12) implies (1.10). If $\alpha$ is supercritical, then $\gamma \geq 0$ and (1.11) holds under the condition (1.12).

\section{References}

[Dyn94] E. B. Dynkin, An introduction to branching measure-valued processes, CRM Monograph Series, 6. American Mathematical Society, Providence, RI, 1994.

[Dy02] _ Diffusions, superdiffusions and partial differential equations, AMS Colloquium Publications, 50. American Mathematical Society, Providence, RI, 2002.

[DK96] E. B. Dynkin, S. E. Kuznetsov, Superdiffusions and removable singularities for quasilinear partial differential equations, Comm. Pure Appl. Math 49 (1996), 125-176.

[DK98] _ Fine topology and fine trace on the boundary associated with a class of semilinear differential equations, Comm. Pure Appl. Math. 51 (1998), 897-936.

[GT98] D. Gilbarg, N.S. Trudinger, Elliptic partial differential equations of second order, Second edition. Springer-Verlag, Berlin-Heidelberg-New York, 1998.

[GrW] M. Grüter, K.-O. Widman, The Green function for uniformly elliptic equations, Manuscripta Math. 37 (1982), 303-342. 
[Le95] J.-F. Le Gall, The Brownian snake and solutions of $\Delta u=u^{2}$ in a domain, Probab. Theory Related Fields 102 (1995), 393-432.

[Maz75] V.G. Maz'ya, Beurling's theorem on a minimum principle for positive harmonic functions, J. Soviet Math. 4 (1975), 367-379.

[Ms02] B. Mselati, Classification et représentation probabiliste des solutions positives de $\Delta u=u^{2}$ dans un domaine, PhD Thesis, Université Paris 6, 2002.

Department of Mathematics, Cornell University, Ithaca, NY 14853, U.S.A.

E-mail address: ebd1@cornell.edu

Department of Mathematics, University of Colorado, Boulder, CO 80309-0395, U.S.A.

E-mail address: Sergei.Kuznetsov@Colorado.edu 